\title{
On the coupling between unstable magnetospheric particle populations and resonant high $m$ ULF wave signatures in the ionosphere
}

\author{
L. J. Baddeley ${ }^{1}$, T. K. Yeoman ${ }^{1}$, D. M. Wright ${ }^{1}$, K. J. Trattner ${ }^{2}$, and B. J. Kellet ${ }^{3}$ \\ ${ }^{1}$ Department of Physics and Astronomy, University of Leicester, University Road, Leicester LE1 7RH, UK \\ ${ }^{2}$ Lockheed Martin ATC, Palo Alto, USA \\ ${ }^{3}$ Rutherford Appleton Laboratory, Didcot, UK
}

Received: 6 July 2004 - Revised: 1 November 2004 - Accepted: 19 November 2004 - Published: 28 February 2005

\begin{abstract}
Many theories state that Ultra Low Frequency (ULF) waves with a high azimuthal wave number $(m)$ have their energy source in wave-particle interactions, yet this assumption has been rarely tested numerically and thus many questions still remain as to the waves' exact generation mechanism. For the first time, this paper investigates the cause and effect relationship between the driving magnetospheric particle populations and the ULF wave signatures as observed in the conjugate ionosphere by quantitatively examining the energy exchange that occurs. Firstly, a Monte Carlo method is used to demonstrate statistically that the particle populations observed during conjugate ionospheric high $m$ wave events have more free energy available than populations extracted at random. Secondly, this paper quantifies the energy transferred on a case study basis, for two classes of high $m$ waves, by examining magnetospheric Ion Distribution Functions, (IDFs) and directly comparing these with the calculated wave energy dissipated into the conjugate ionosphere. Estimates of the wave energy at the source and the sink are in excellent agreement, with both being of the order of $10^{10} \mathrm{~J}$ for a typical high $m$ wave. Ten times more energy $\left(10^{11} \mathrm{~J}\right)$ is transferred from the magnetospheric particle population and dissipated in the ionosphere when considering a subset of high $m$ waves known as giant pulsations (Pgs). Previous work has demonstrated that $10^{10} \mathrm{~J}$ is frequently available from non - Maxwellian IDFs at $\mathrm{L}=6$, whereas $10^{11} \mathrm{~J}$ is not. The combination of these studies thus provides an explanation for both the rarity of Pgs and the ubiquity of other high $m$ waves in this region.
\end{abstract}

Key words. Magnetospheric physics (MHD waves and instabilities) - Space plasma physics (Transport processes; Wave-particle interactions)

Correspondence to: L. J. Baddeley

(lisa.baddeley@ion.le.ac.uk)

\section{Introduction}

This paper will examine standing mode waves on closed magnetic field lines in the Earth's inner magnetosphere. Such Ultra Low Frequency (ULF) waves are vital in transferring and distributing energy and information throughout the magnetosphere-ionosphere system. The governing MHD wave equations in a dipolar field (e.g. Dungey, 1963) allow the waves to be classified into two basic categories with respect to their generation mechanism. The first type, toroidal modes, has an electric field polarization in the radial direction and is characterised by the small magnitude of their effective azimuthal wave numbers, $m$ (or equivalently their large-scale size in the azimuthal direction). These waves generally have an energy source external to the Earth's magnetosphere and can include such mechanisms as the KelvinHelmholtz instability on the magnetopause, solar wind buffeting or an impulsive increase in solar wind pressure at the front of the magnetopause. The second type of waves, poloidal modes, has an electric field polarisation in the azimuthal direction and is conversely characterised by their high magnitude $m$ numbers and small azimuthal scale sizes. It is these small-scale waves which are the focus of this paper. The generation mechanism thought to be responsible for these small-scale waves is internal to the magnetosphere, taking the form of wave-particle interactions with non-Maxwellian or unstable particle populations in the global ring current. These particle populations contain "free energy" which is extracted by a resonant wave field and ultimately dissipated into the ionosphere by the wave electric field through Joule heating (e.g. Newton et al., 1978). Theoretically, a great deal of work has been undertaken in examining this interaction mechanism (e.g. Hasegawa, 1971; Southwood, 1976; Cheng 1982; Kivelson and Southwood, 1985; Pokhotelov et al., 1986; Cheng and Qian, 1994). However, the hypothesis that the waves are driven in this manner has rarely been experimentally tested (the authors are aware of only four documented cases in total; Hughes et al., 1978; Glassmeier et al., 1999; Wright et al., 2001 and Baddeley et 
al., 2002 (data from the latter two papers are utilised in this paper). Additionally, the energy that is transferred from the magnetospheric particle populations into the ionosphere has never been documented.

There are many reasons as to the lack of conclusive observational evidence to validate theories concerning high $m$ waves. Firstly and primarily, the nature of large-scale waves is such that they are difficult to observe using ground magnetometers and also satellites. When considering ground observations, such factors as limited instrumentation coverage and spatial and temporal resolution must be considered. The ground magnetic field is dominated by the effect of the Eregion Hall currents. Ground magnetometers work by averaging information from an ionospheric region whose scale size is similar to the height of the E-region. This act of spatial integration introduces problems when observing high $m$ waves which have large phase variations over a limited spatial extent, which means that ground magnetometer data alone are far from optimal for this study. ULF waves are observed by both VHF and HF radars, providing higher spatial resolution $(15-45 \mathrm{~km})$ in the E- and F-region ionospheres, respectively, although any large-scale convection flow that is present can mask the wave signature due to the small velocity perturbation of the wave field.

Satellites traversing an active region in the magnetosphere, often at great speed, are also not always the optimal instrument, and as such can often give an incomplete picture of the occurrence of highly localized, large-scale waves.

The most accurate method of determining a direct cause and effect relationship between the driving particle population and a high $m$ wave, as well as ascertaining the energy transferred in any interaction, is through conjugate groundsatellite observations. The problems outlined above make this type of study most difficult. However, new instrumentation, such as the DOppler Pulsation Experiment (DOPE, Wright et al., 1997) and new observational techniques such as the artificial backscatter technique (e.g. Yeoman et al., 1997), allow for more detailed observations of large-scale waves in the ionosphere. Here we utilise the combination of satellite observations and such novel ground-based instrumentation. A statistical test of the assumption that high $m$ ULF waves are indeed related to unstable particle populations in the magnetospheric ring current has been undertaken using conjugate measurements of driving magnetospheric particle populations made by the Polar spacecraft and ionospheric wave observations made by the DOPE HF sounder. Specifically, this method is employed to test if the particle populations observed during conjugate ionospheric high $m$ wave events have more free energy available than populations extracted at random from the database. In addition, we quantify, for the first time, the amount of energy transferred during the interaction by examining the energy available to the wave from the collisionless plasma of the ring current and comparing this to the energy dissipation due to the wave in the collisional ionospheric plasma. These calculations are undertaken for two case events of typical classes of high $m$ wave; (1) a wave that displays no corresponding ground mag- netometer signature, a so-called uncorrelated wave (UW), and (2) a giant pulsation or Pg, which, due to its large amplitude and modest azimuthal wave number, does have a corresponding ground magnetic signature.

As mentioned above, instrument limitations have meant that the amount of energy that is transported through the magnetosphere - ionosphere system by large-scale waves has been difficult to quantify. The combination of the statistical and case studies presented here helps to shed light on this process and also serves to reinforce the importance of largescale ULF waves when considering how solar wind energy enters into and affects the Earth's ionosphere.

\section{Instrumentation}

\subsection{DOppler Pulsation Experiment (DOPE)}

The DOPE HF Sounder, located near Troms $\varnothing$, Norway has been operational since May 1995 and is designed to make measurements of ionospheric signatures of ULF waves at high latitudes (see Wright et al., 1997 for a more detailed instrument description). The excellent ionospheric spatial resolution (of the order of 3-4 km for F-region reflection height, Wright et al., 1997) and continuous operation of the instrument gives unprecedented observations of high $m$ waves. Additionally, the location of the instrument is such that the observed waves are on field lines which map to the equatorial ring current, the major energy source for such pulsations. The data used here were obtained when the system had a configuration of one frequency-stable transmitter and a receiver with a ground separation of $50 \mathrm{~km}$. While this original DOPE system still provided an excellent tool for detecting large-scale waves in the ionosphere, the lack of knowledge about the spatial scale of the wave in azimuth, due to the fact that only two paths (O-mode signal and $\mathrm{X}$-mode signal) in the same meridian (which were height separated) were in operation, meant that the $m$ numbers of the waves were not directly measured.

Wright et al. (1999) and Yeoman et al. (2000) have presented several observations of ULF waves observed by the DOPE HF sounder. In their papers they demonstrate the ability of the DOPE HF sounder to observe large-scale ULF waves that would be unseen by ground magnetometer data. Yeoman et al. (2000) indicate that a significant population of high $m$ waves (" $m$ of the order of 100") were observed in the morning sector ionosphere while Wright et al. (1999) demonstrate populations of high $m$ waves in both the dawn and dusk sectors. Given the evidence presented in these two extensive studies, we are confident that the observations presented in this paper are indeed those of large-scale waves.

\subsection{Polar spacecraft}

The Polar spacecraft was launched on 24 February 1996 with the objective of making both high- and low-altitude measurements of the Earth's polar regions. 




Fig. 1. Schematic indicating how the free energy contained in the positive gradient region is calculated. The point A represents the location in phase space where the particles which contain free energy are transposed to.

The spacecraft describes a highly elliptical orbit, with apogee at 9 Earth radii $\left(\mathrm{R}_{E}\right)$, perigee at $1.8 \mathrm{R}_{E}$ geocentric and an inclination of $86^{\circ}$. The orbital period is about $18 \mathrm{~h}$ with a spacecraft spin period of $6 \mathrm{~s}$. Initially apogee was over the northern polar region, but apogee has been moving towards the equator at about $18^{\circ}$ per year. This highly elliptical orbit of Polar allows the spacecraft to sample the inner magnetospheric particle populations, more specifically, populations located in the ring current thought to drive the ULF waves. Additionally, the precession of the orbit also allows the entire magnetosphere to be sampled over time. The spacecraft spin axis is normal to the orbital plane to allow a despun platform for the auroral imagers and to enable the particle instruments to map out the full $4 \pi$ steradian distribution functions. The TIMAS instrument (Shelley et al., 1995) measures the lower energy protons from $\sim 0.025 \mathrm{keV}$ to $32 \mathrm{keV}$, while the CAMMICE (Wilken et al., 1992) instrument extends to higher energies, $1 \mathrm{keV}$ to $328 \mathrm{keV}$.

\section{Statistical analysis of wave and particle distribution function occurrence}

\subsection{Energy available from the driving particle population}

To determine if a particle population can induce wave growth or damping, its Ion Distribution Function (IDF), $f$, must be analysed as a function of particle energy, $W$. Free energy, which manifests itself as a positive gradient region, $\mathrm{d} f / \mathrm{d} W>0$, a bump-on-tail on the IDF, which elsewhere follows a monotonic power law (Fig. 1), is fed into the wave mode through a process of inverse Landau damping. Here, such bump-on-tail distributions were identified by the
TIMAS and CAMMICE (MICS) instruments. Only data points within the positive gradient region were examined, as illustrated in Fig. 1. Each energy bin has an upper and lower limit, $W_{u}$ and $W_{l}$, with an average value for the particle energy in that bin of $W_{i}=\left(W_{u}+W_{l}\right) / 2$. Each data point $W_{i}$ has a corresponding average distribution function in velocity space $\partial \mathrm{f}_{i}$. Each $\partial \mathrm{f}_{i}$ was then transformed back from velocity space to real space, to determine the average number density of particles, $\partial n_{i}$, at that particular energy. It was then assumed that all particles with free energy (i.e. in the positive gradient region) would return to some predefined minimum, ( $W_{\min }$, $f_{\min }$ ) which is set by the location of the initial point of the positive gradient region in each particle distribution (marked point A on Fig. 1). This can be thought of as a conservative estimate as to the energy release needed for the population to return to a completely monotonic distribution which is given by

$E=\sum_{i}\left(\partial n_{i}-\partial n_{\min }\right)\left(W_{i}-W_{\min }\right)$.

By numerical integration of a dipole field an estimate can be made of the corresponding flux tube volume, $V$, which the particle population occupies and thus the total free energy available in Joules to a possible resonant wave on that flux tube can be quantified,

$E_{\text {total }}=V * \sum_{i}\left(\partial n_{i}-\partial n_{\min }\right)\left(W_{i}-W_{\min }\right)$.

The dipole geometry is such that the flux tube volume is dominated by the equatorial regions. A more complete approach would require knowledge of individual particle trajectories in velocity space. This is beyond the scope of this study, which provides a conservative estimate of the available free energy. A more detailed discussion of this calculation can be found in Baddeley et al. (2005).

\subsection{Particle population database}

The database used in both the Monte Carlo study and also the case studies presented later in this paper comprises of $\sim 2500$ IDFs amassed over a 2.5-year period from May 1996 to December 1998, as measured by both the TIMAS and CAMMICE(MICS) instruments on board the Polar spacecraft. Each IDF was measured simultaneously by both the lower energy range detector, TIMAS, and the higher energy range detector, CAMMICE (MICS). Table 1 shows the energy bin numbers and energy range of each bin for the TIMAS instrument. The overlap in energy ranges of the two instruments (see Sect. 2.2) provides confidence in the resulting structure of the IDF. All IDFs contained in the database were measured only when the spacecraft was traversing the ring current (L-shell locations of between 6 and 9). In all there are $\sim 100$ IDFs in each hourly MLT bin from 07:00 16:00 and 18:00-06:00 MLT, with $\sim 70$ data points in each bin in the early morning and late evening sectors. In all numerical calculations, TIMAS data were utilised when considering particle energies of $\leq 21 \mathrm{keV}$, as this instrument has a higher energy resolution than CAMMICE (MICS) at these lower energies. 
Table 1. The energy bin numbers and energy ranges in keV for the TIMAS particle detector on board the Polar spacecraft which were used in the calculation of the free energy and slope of the positive gradient region in the text.

\begin{tabular}{cc}
\hline Channel Number & Energy $(\mathrm{keV})$ \\
\hline 14 & 2.0 \\
15 & 2.438 \\
16 & 2.972 \\
17 & 3.623 \\
18 & 4.416 \\
19 & 5.384 \\
20 & 6.653 \\
21 & 8.0 \\
22 & 9.752 \\
23 & 11.89 \\
\hline
\end{tabular}

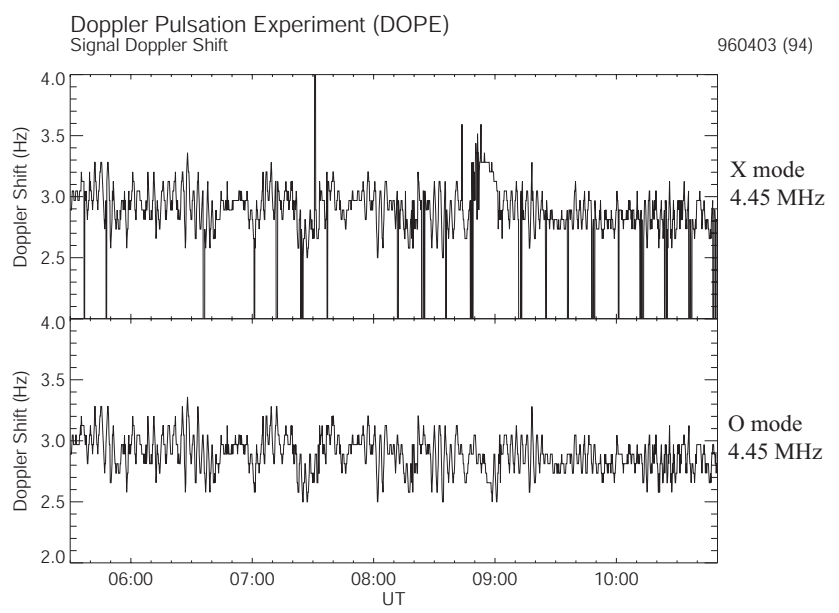

Fig. 2. An example of a Doppler trace using the original 2 beam DOPE system. A ULF wave signature is seen in both channels, although predominantly in channel 2 (O mode), between 05:30 to 10:30 UT. The DOPE sounding frequencies are shown to the right of each trace. Both traces show unfiltered data and as such there are data spikes, probably due to HF noise, in panel (a).

\subsection{ULF wave database}

The conjugate ground-satellite study presented here utilises the single path DOPE system. This was due to fact that from October 1998 the data from the TIMAS particle instrument on board Polar became unreliable. During this time, from May 1995 to October 1998, DOPE measured 130 events. An example of a Doppler trace utilised in the study is shown in Fig. 2. The sounding frequency for the two height separated traces are shown next to each plot. Both traces indicate the presence of a ULF wave from 05:30 to 10:30 UT. Both traces show unfiltered data and as such there are data spikes, probably due to HF noise, in panel (a).
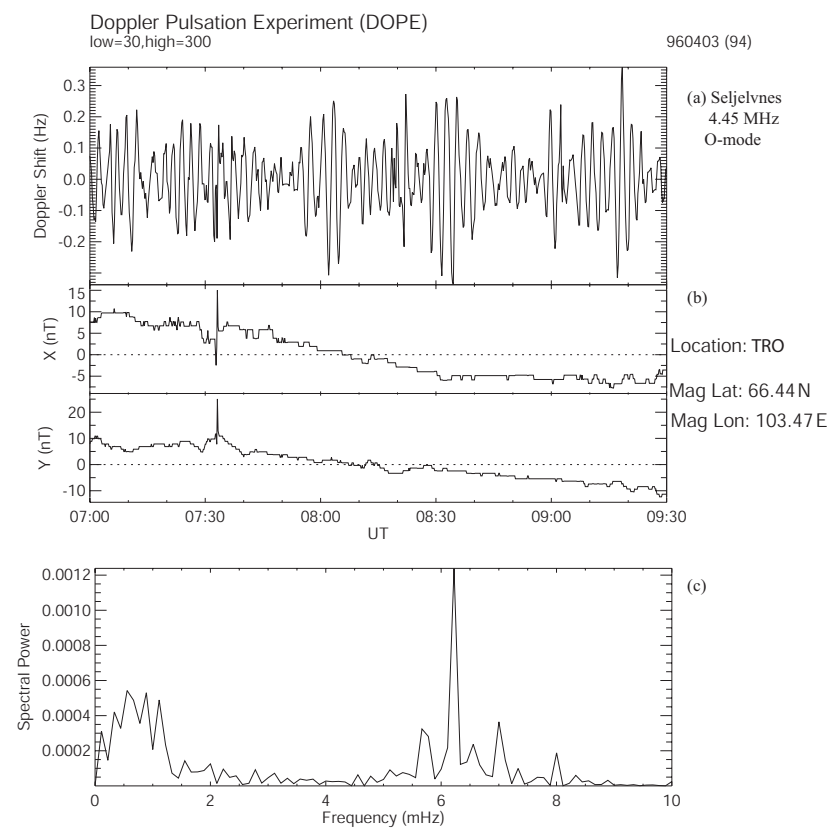

Fig. 3. (a) The filtered O mode DOPE trace from Fig. 2 and (b) conjugate ground magnetometer data ( $\mathrm{X}$ and $\mathrm{Y}$ components) from the TRO magnetometer. Panel (c) indicates results from a spectral analysis of the DOPE data and indicates the presence of a $6-\mathrm{mHz}$ wave in the ionosphere. The wave is classed as uncorrelated, as there is no signature of it in the magnetometer data.

\subsection{Statistical analysis}

A spectral analysis was undertaken on ground magnetometer data to ascertain whether the wave displayed a conjugate ground signature. An example of this analysis is shown in Fig. 3. The filtered Doppler data from Fig. 2 is shown in panel (a). The data from channel 2, presented in Fig. 2, have been filtered between $30 \mathrm{~s}$ and $300 \mathrm{~s}$ to remove the data spikes. Conjugate magnetometer data from the TRO magnetometer are also presented. The results of the spectral analysis undertaken on the DOPE data are shown in panel (c). Figure $3 \mathrm{c}$ indicates that the wave observed by DOPE had a frequency of $\sim 6 \mathrm{mHz}$. The wave is classed as uncorrelated, as there is no evidence of a $6-\mathrm{mHz}$ wave in the magnetometer data. All the waves in this study displayed no conjugate high $m$ magnetic signature on the ground. One event did display a possible harmonically related magnetic ground signature at a lower frequency to that observed by DOPE. This wave was still classed as uncorrelated, as there was no direct frequency signature observed in the magnetometer data at the same frequency to that observed in the ionosphere. The databases were examined to obtain instances where a ULF wave event which displayed no conjugate ground magnetometer signature was observed by DOPE for which conjugate particle data were available from Polar. A window of $\pm 1.5 \mathrm{~h}$ in UT of conjugacy was allowed between the 2 databases for each event.

This amalgamation of databases resulted in only 6 wave events observed by DOPE for which particle data were 



Fig. 4. Panels (a) to (f) - the 6 IDFs measured by the TIMAS and CAMMICE (MICS) instruments for which there were conjugate ionospheric wave observations from the DOPE HF sounder. Panel $(\mathbf{g})-$ An example of an IDF taken from the modal bin $\left(0.0\right.$ to $\left.5.6 \times 10^{9} \mathrm{~J}\right)$ of the Monte Carlo distribution shown in Fig. 6a. The IDF contains a positive gradient region in the form of a "shoulder" in the distribution.

available. In 5 cases the events were exactly conjugate, with Polar passing through the same MLT location at the same UT as the wave event. In one case Polar observed the particle population at a location eastward of DOPE at an earlier UT. This event can still be considered as conjugate as the particles would be expected to drift westward from that location to lie over Troms $\varnothing$ at the time of the wave event. Figures $4 \mathrm{a}-\mathrm{f}$ shows the 6 IDFs for which a wave was observed at the conjugate point in the ionosphere. The following text will refer to these 6 IDFs as the conjugate IDFs. The conjugate IDFs all contain a positive gradient region between particle energies of $\sim 2 \mathrm{keV}$ and $\sim 12 \mathrm{keV}$, and were measured when Polar was located between 09:00 and 16:00 MLT. For purposes of a statistical significance test the conjugate IDFs were compared to a database of IDFs measured between this MLT range. This new database contained 792 IDFs and was characterised by the gradient of the IDF between $2 \mathrm{keV}$ and $12 \mathrm{keV}$. Only TIMAS data were utilised in this calculation, as this instrument has a higher energy resolution than CAMMICE (MICS) at energies $\leq 12 \mathrm{keV}$. The gradient of the distribution was used to characterise the database instead of the free energy that each IDF possessed, as not all IDFs possess free energy. This distribution is shown in Fig. 5. The $\mathrm{x}$-axis represents the gradient of the distribution, from $-10000 \mathrm{~km}^{-6} \mathrm{~s}^{3} \mathrm{keV}^{-1}$ to $10000 \mathrm{~km}^{-6} \mathrm{~s}^{3} \mathrm{keV}^{-1}$, with the data binned in bin sizes of $100 \mathrm{~km}^{-6} \mathrm{~s}^{3} \mathrm{keV}^{-1}$. The $\mathrm{y}$-axis indicates the number of occurrences in each of these bins. The overall nature of the distribution appears to be bell shaped, with a calculated mean value of $-277.7 \mathrm{~km}^{-6} \mathrm{~s}^{3} \mathrm{keV}^{-1}$ but with a long tail towards highly negative gradient values. This tail in the distribution resulted in a calculated value for the standard deviation of $8004.5 \mathrm{~km}^{-6} \mathrm{~s}^{3} \mathrm{keV}^{-1}$, which meant a statistical test, such as 


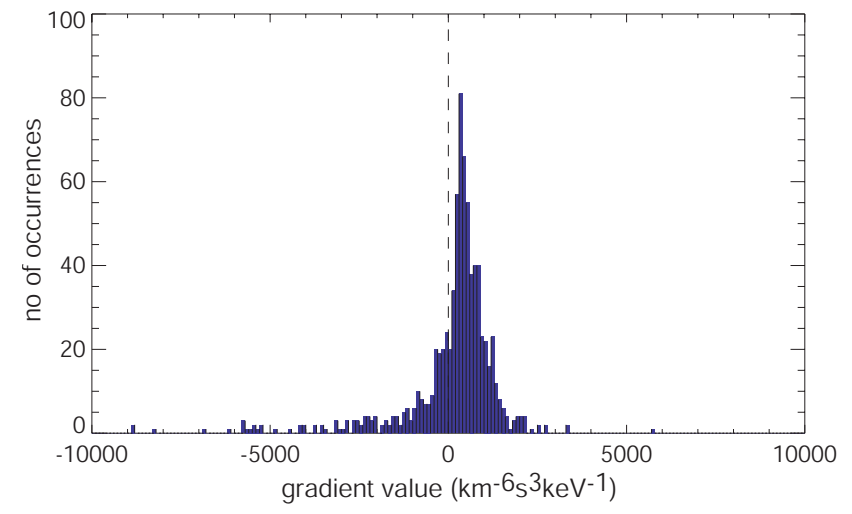

Fig. 5. Distribution of the gradient magnitude measured between $\sim 2 \mathrm{keV}$ and $12 \mathrm{keV}$ for all IDFs measured between 09:00 and 16:00 MLT.

a student t-test, was deemed unsuitable. Given the shape of this distribution it was decided that a Monte Carlo analysis, utilising the amount of free energy, would provide a more suitable statistical test as to the significance (if any) of the conjugate IDFs. The free energy possessed by each of the conjugate IDFs was compared to that of 6 IDFs extracted from the database at random. The distribution of the free energy of these 6 random IDFs was plotted and the procedure was repeated 10000 times. The resulting probability distribution for the amount of free energy is shown in Fig. 6a. The $\mathrm{x}$-axis indicates the free energy available from an IDF. The blue (Monte Carlo) distribution is the mean free energy distribution of 6 randomly extracted IDFs. The yellow distribution is the free energy distribution for the conjugate IDFs. An example of an IDF taken from the modal bin (0.0 to $5.6 \times 10^{9} \mathrm{~J}$ ) of the Monte Carlo distribution is shown in Fig. 4g. The IDF contains a positive gradient region in the form of a "shoulder" in the distribution and contains a reduced amount of free energy when compared to the majority of conjugate IDFs.

The Monte Carlo analysis was also utilized to examine the average amount of free energy possessed by the conjugate IDFs compared to the average amount possessed by 6 IDFs randomly extracted from the database. Using the method outlined earlier, the average free energy available from the conjugate IDFs was calculated to be $\sim 3 \times 10^{10} \mathrm{~J}$. In the case where a randomly-chosen IDF possessed no free energy in the form of a positive gradient region between 2 and $12 \mathrm{keV}$, a value of $0 \mathrm{~J}$ was recorded. The operation was again performed 10000 times. The results are displayed in Fig. 6 b. The average free energy possessed by 6 randomly extracted IDFs is shown on the $\mathrm{x}$-axis in bin sizes of $6 \times 10^{9} \mathrm{~J}$, with a probability for each bin shown on the y-axis. The average free energy available from the 6 IDFs for which a wave event was recorded $\left(\sim 3 \times 10^{10} \mathrm{~J}\right)$ is marked with a red vertical dashed line.

This analysis thus indicates that the average free energy available from a particle population during times when a high $m$ wave is observed in the conjugate ionosphere is greater
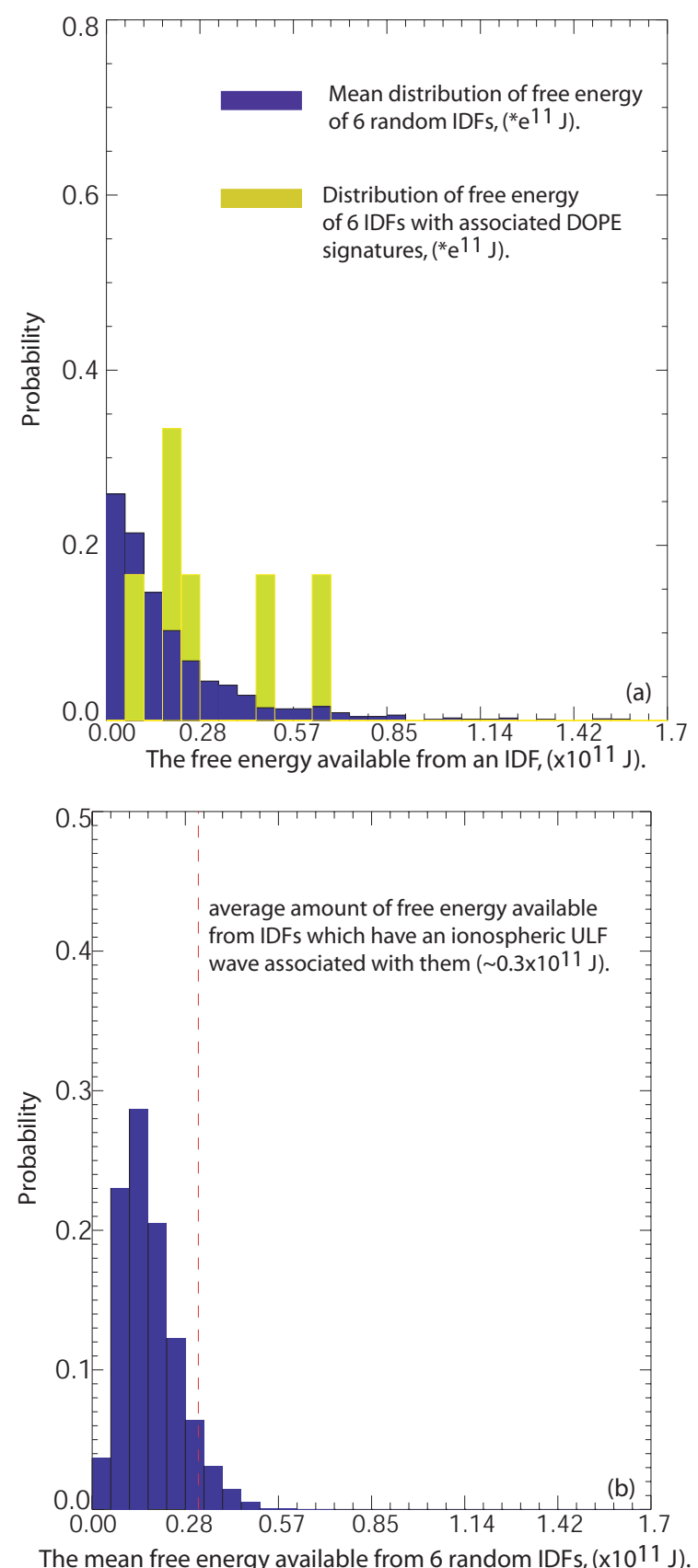

Fig. 6. The Monte Carlo analysis results for the two tests described in the text. Panel (a) shows the probability distribution for the amount of free energy of 6 random IDFs. The $x$-axis indicates the free energy available from an IDF. The blue (Monte Carlo) distribution is the mean free energy distribution of 6 randomly extracted IDFs. The yellow distribution is the free energy distribution for the conjugate IDFs. Panel (b) shows the distribution of the average free energy available from a sample of 6 random IDFs extracted from the database described in this paper. The average free energy for the 6 IDFs for which a conjugate ionospheric ULF wave was detected by DOPE is marked with a vertical red dashed line. A valve of $93.6 \%$ of the distribution lies below this red line, indicating a statistical cause and effect relationship between the driving particle populations and the ULF waves. 
than that seen in a random sample, and that this difference is statistically significant, with $93.6 \%$ of the distribution located below this value. Despite the small number of events used in this study, this is good evidence of a genuine causal relationship between the gradient magnitude and resulting free energy contained in the IDF, and the occurrence of a high $m$ wave being observed in the conjugate ionosphere.

\section{Case studies of morning sector particle-driven high $m$ ULF waves}

In this section two case studies are presented where a ULF wave was observed in the ionosphere and the driving particle population was also observed in the magnetosphere, under circumstances where an estimate could be made of both the energy contained in the wave source (the particle population) and in the wave energy sink (Joule heating of the ionosphere).

Although ground-satellite observations have been previously documented (the authors are aware of only three documented cases in total; Glassmeier et al., 1999; Wright et al., 2001 and Baddeley et al., 2002, the latter two events are presented here), this paper presents the first direct comparison using the amount of energy that is transferred from the particle population into the ionosphere by the wavefield. In both cases used in this paper the resonance condition (Southwood et al., 1969),

$\omega_{\text {wave }}-m_{\text {wave }} \omega_{\text {drift }}=N \omega_{\text {bounce }}$

was satisfied, where $N$ is an integer (usually \pm 1 or 0 ) and $\omega_{\text {wave }}, \omega_{\text {bounce}}$, and $\omega_{\text {drift }}$ are the angular frequencies of the wave, the proton bounce, and the proton azimuthal drift, respectively. This condition allows the driving particles to be phase locked with that of the wave field, thus allowing energy transfer to occur. The type of interaction and particle energies involved in the process depend upon the standing mode structure of the wave.

\subsection{Analysis procedure}

The theory briefly outlined in Sect. 3.1 was used to calculate the free energy possessed by the driving magnetospheric particle population, which is potentially available as a source of wave energy. To quantify the energy dissipated in the ionosphere, the ultimate energy sink, the method employed by Greenwald and Walker (1980) is used here. Briefly, there exists a balance between the magnitude of the averaged downward wave Poynting flux into the ionosphere and the Joule dissipation rate within it;

$\frac{1}{\mu_{0}} E b=J_{P} E=\Sigma_{P} E^{2}$,

where $\mu_{0}$ is the permeability of free space, $J_{P}$ is the Pedersen current, $\Sigma_{P}$ is the height-integrated Pedersen conductivity, E is the magnitude of the wave electric field and $b$ is the magnitude of the wave magnetic field. The total energy dissipated in Joules into the ionosphere, $P$, can then be quantified by,

$P=2 A \Sigma_{P} E^{2} T_{1}$

where $A$ is the area of dissipation in the ionosphere, typically $1^{\circ}$ latitude by $5^{\circ}$ of longitude for a high $m$ wave at $L \sim 6$, (e.g. Baddeley et al., 2002) and $T_{1}$ is the duration of the wave. The factor of 2 arises from the fact that the wave energy is dissipated simultaneously into both the Northern and Southern Hemisphere ionospheres. In the calculations for the wave sink and source, the area of ionospheric dissipation and the flux tube volume calculations were identical, thus eliminating errors due to any potential underestimate of the spatial extent of the wave. In both the waves considered in this paper ground observations were made at Troms $\varnothing$; an $L$-shell location of $\sim 6.3$. Here we have chosen two examples of high $m$, particle-driven waves. The first is a high $m$ wave observed by both the CUTLASS and EISCAT radars in an experiment utilising the heating facility at the EISCAT Troms $\varnothing$ site (see Baddeley et al., 2002). The second is a Pg observed by the DOPE system and the IMAGE magnetometer array (see Wright et al., 2001). In both cases conjugate particle data from Polar particle instruments were available.

\subsection{Case study 1 - A high $m$ wave observed on 26 October 1998}

The details of the experimental setup and a full analysis of this wave event are available in Baddeley et al. (2002). The Pc4 wave in question had a measured frequency of $\sim 14 \mathrm{mHz}$, an azimuthal wave number, $m$ of -45 and was observed using the CUTLASS Hankasalmi radar in artificially generated backscatter during a special mode from 08:40-09:40 UT (10:40-11:40 MLT) on 26 October 1999. Figure 7a indicates the line of sight velocities for individual range gates, (27-29) for beam 5 of the radar as a stacked time series. Figures $7 \mathrm{~b}$ and $\mathrm{c}$ indicate the $\mathrm{X}$ and $\mathrm{Y}$ magnetic field components, as measured by the IMAGE magnetometer at Troms $\varnothing$ and reveal that the wave has no associated ground signature. The CUTLASS Hankasalmi and EISCAT Troms $\varnothing$ radars were utilized to obtain values for the ionospheric electric field, E, $\left(5 \mathrm{mV} \mathrm{m}^{-1}\right)$ and the height integrated Pedersen conductivity, $\Sigma_{P}$ (3 mho). By inserting these measured values for $\Sigma_{P}$ and E into Eqs. (4) and (5) it can be calculated that $\sim 10^{10} \mathrm{~J}$ of energy was dissipated into the ionosphere during this wave event, at a dissipation rate of $7.5 \times 10^{-5} \mathrm{~W} \mathrm{~m}^{-2}$. Figure $7 \mathrm{~d}$ shows conjugate particle data for this event from the CAMMICE (MICS) instrument. This particle data revealed that free energy was available in the form of a positive gradient region at $10 \mathrm{keV}$. Values for the wave frequency $(14 \mathrm{mHz})$ and $m$ number $(-45)$ obtained using the radar data, and the correct angular drift and bounce frequencies for a $10 \mathrm{keV}$ proton population were input into Eq. (3). The equation could be solved for an $N=1$, drift bounce resonance interaction, indicating the wave had a second harmonic standing mode structure. 


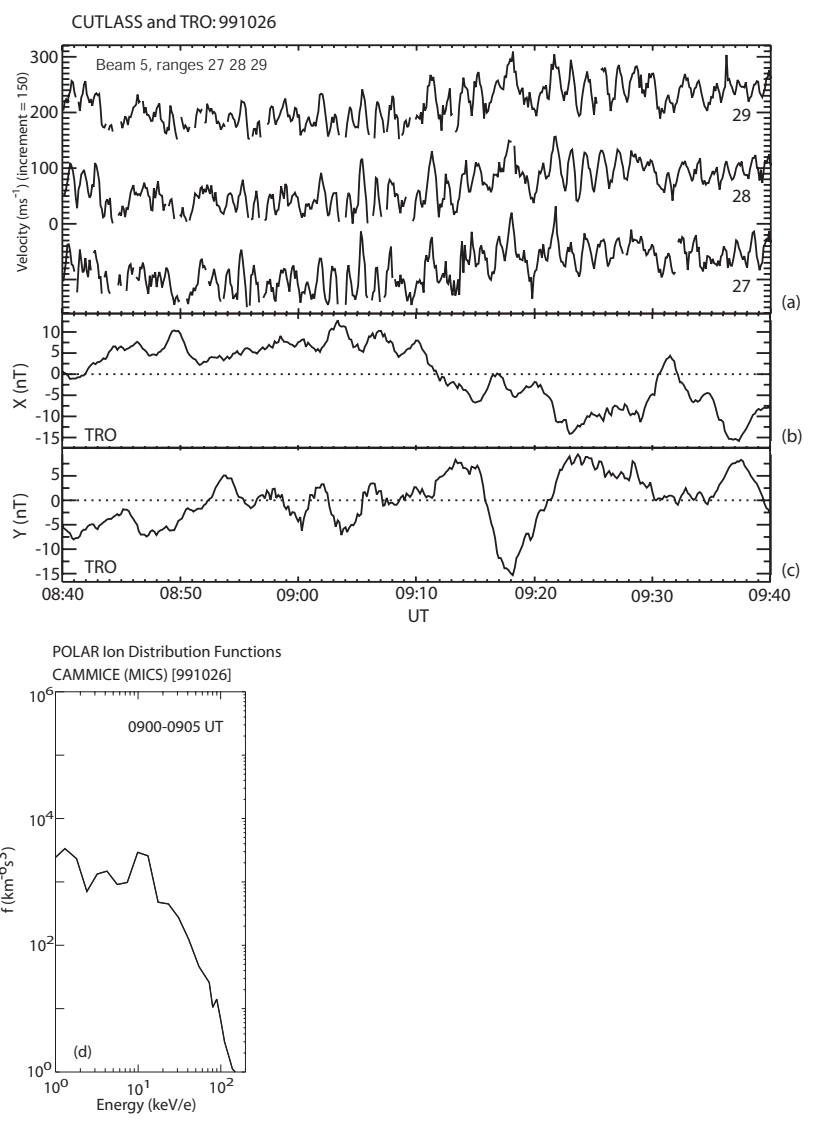

Fig. 7. (Taken from Baddeley et al., 2001, described as case study 1 in this paper) Data from 26 October 1999, (a) CUTLASS stack plot for beam 5, range gates 27-29. (b) and (c) Corresponding measurements of the ground magnetic field at Troms $\varnothing$, for both the $\mathrm{X}$ and $\mathrm{Y}$ components. (d) The conjugate driving ion distribution functions, IDFs, derived from the CAMMICE (MICS) instrument for the interval on 26 October 1999, 09:00:00-09:05:00 UT.

Calculations utilizing Eq. (2) revealed that in this case there was $\sim 10^{10} \mathrm{~J}$ of free energy available to the wave from the driving magnetospheric particle population.

\subsection{Case study 2 - giant pulsation observed on 16 May 1998}

The details of the experimental setup and a full analysis for this event are available in Wright et al. (2001). The Pg had a measured frequency of $\sim 6 \mathrm{mHz}$ and an $m$ number of $\sim-30$ and was observed using the DOPE system (Figs. 8a and b on 16 May 1998 commencing at $~ 06: 00$ UT (08:00 MLT), lasting for approximately $3.5 \mathrm{~h}$. Measurements made by the IMAGE network of magnetometers (Figs. $8 \mathrm{c}$ and d) revealed that the wave was of large enough amplitude to have a magnetic ground signature, most prominently in the $\mathrm{Y}$ component, with the peak amplitude of the magnetic oscillations occurring south of Troms $\varnothing$. Unfortunately for this event, direct measurements of the electric field and height integrated Pedersen conductivities were unavailable. DOPE measurements imply an electric field of $8 \mathrm{mVm}^{-1}$ at Troms $\varnothing$ (see

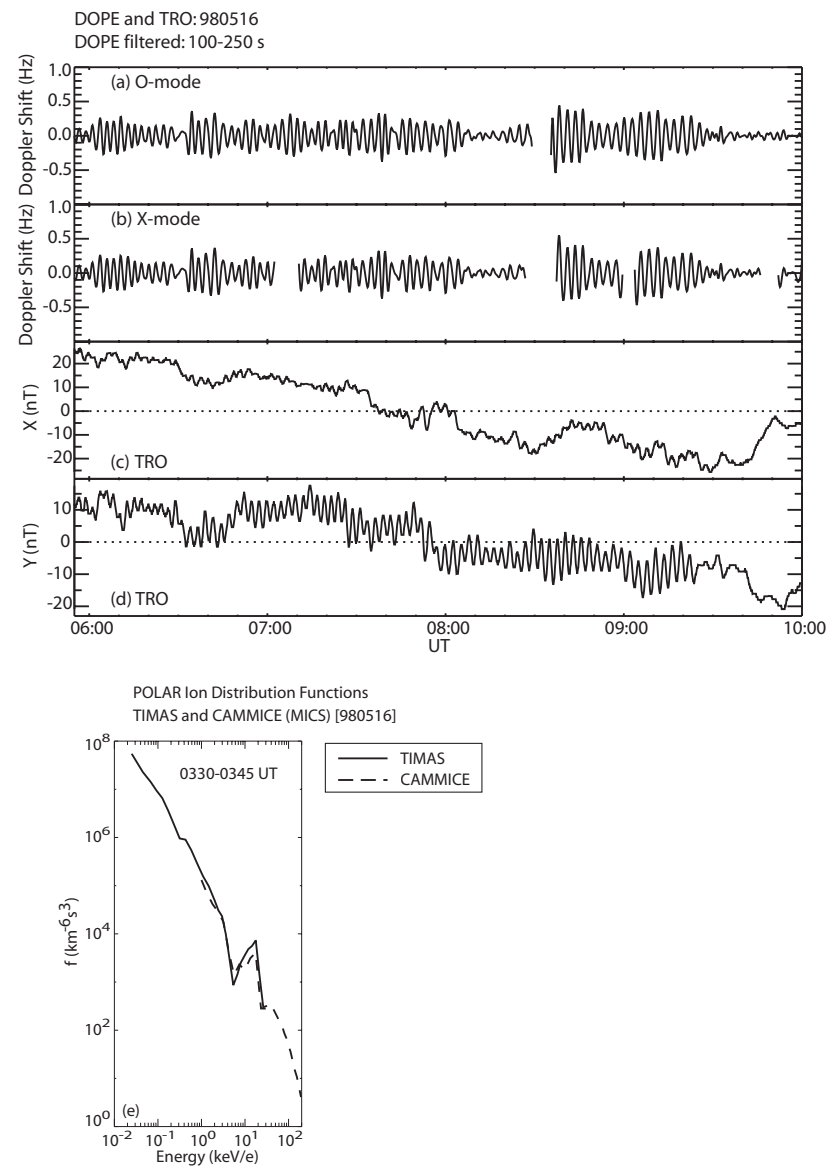

Fig. 8. (Taken from Wright et al. 2001, described as case study 2 in this paper) (a) O- and (b) X- mode, HF Doppler sounder data from DOPE and (c) $\mathrm{X}$ and (d) Y component ground magnetometer data at Troms $\varnothing$ for 06:00-10:00 UT on 16 May 1998 during the occurrence of a giant pulsation $(\mathrm{Pg})$. (e) The conjugate driving ion distribution functions, IDFs, derived from the TIMAS and CAMMICE (MICS) instruments for the interval on 16 May 1998, 03:30:00-03:45:00 UT.

Wright et al., 1998 for details of the technique employed). Magnetometer data indicate that the amplitude peak of the pulsation is located to the south of Troms $\emptyset$ and suggests an electric field of $15 \mathrm{mVm}^{-1}$ at the Pg peak. Similar values were obtained by the SABRE radar (Chisham et al., 1992) in one of only two radar detections of Pg pulsations ever recorded. This value is three times that measured for the wave presented in Sect. 4.2. A typical value of the height integrated Pedersen conductivity was also used, $\Sigma_{P} \sim 3$ mho. Using Eqs. (4) and (5) it was revealed that for this larger amplitude wave $\sim 10^{11} \mathrm{~J}$ of energy was dissipated into the ionosphere at a local dissipation rate of $6.8 \times 10^{-4} \mathrm{~W} \mathrm{~m}^{-2}$, ten times more than that for the wave presented in Sect. 4.2. Figure 8e shows conjugate particle data for this event from both the TIMAS and CAMMICE (MICS) instruments. The particle data revealed that free energy was available in the form of a positive gradient region at $\sim 7 \mathrm{keV}$. 
Values for the wave frequency $(6 \mathrm{mHz})$ and $m$ number $(-30)$ obtained using the DOPE data and the correct angular drift and bounce frequencies for a $7 \mathrm{keV}$ proton population were input into Eq. (3). The equation could be solved for an $N=1$, drift bounce resonance interaction, indicating the wave had a second harmonic standing mode structure. Calculations using Eq. (2) revealed that in this case there was $\sim 10^{11} \mathrm{~J}$ of free energy available to the wave from the driving magnetospheric particle population.

\section{Discussion}

To date a statistical study of high $m$ ULF wave occurrence and conjugate magnetospheric particle populations has never been undertaken, and hence a statistical cause and effect relationship between the particles and waves has been uncertain. This study provides the first statistical experimental evidence that high $m$ waves are driven by internal unstable plasma populations. Despite the small number of events used in this study, there is good evidence of a genuine relationship between the gradient magnitude and resulting free energy contained in the IDF and the occurrence of a high $m$ wave being observed in the conjugate ionosphere. The analysis indicates that the average free energy available from a particle population during times when a high $m$ wave is observed in the conjugate ionosphere is greater than that seen at random, and that this difference is statistically significant, with $93.6 \%$ of the distribution located below this value. This implies that the waves occurred during periods when there was a particle distribution present in the magnetosphere at a conjugate location, with increased free energy available. This result also adds statistical weight to the original theories of internally driven waves (e.g. Southwood et al., 1969; Hasegawa, 1969) and supports the notion that high $m$ waves have an energy source internal to the magnetosphere, in the form the magnetospheric ring current.

For the first time, the independent calculations for the wave energy source and sink for morning sector high $m$ waves shown above demonstrate that $\sim 10^{10} \mathrm{~J}$ of energy is available for transfer from the magnetospheric particle population and subsequent dissipation in the ionosphere in a typical high $m$ wave, whilst $\sim 10^{11} \mathrm{~J}$ is available for a $\mathrm{Pg}$. This energy is available for transfer into the ULF wavefield should the particle energies at which it occurs and the eigenfrequency of the field line upon which it occurs provide a solution to the drift-bounce resonance condition, Eq. (3). In the event that the energetic particles providing the positive gradient region were injected onto the dipolar geomagnetic field lines in a short-lived injection process, then the energy calculated above will be the total energy available to the wave. The magnetospheric region which contains this energy will then drift westwards with the particles themselves. Such a westward drift has been observed in Pgs, (e.g. Glassmeier, 1980; Kokubun et al., 1989; Chisham et al., 1992). Such particles may indeed circumnavigate the Earth and drive a second wave when the drift-bounce reso- nance condition is again met (Chisham, 1996). In the event that the positive gradient region results from the energy dependence of the gradient-curvature drift speed of particles injected over a longer time scale, or from gradient curvature drift alone, then additional energy may be available as the positive gradient region of the IDF is replenished by new particles arriving from the east. In this case the energies above represent a minimum energy available to the wave, and the magnetospheric region containing free energy would remain at a constant local time.

Once transferred to the wave field, the dominant energy dissipation mechanism is through Joule heating at the foot of the field line in the collisional medium of the ionosphere, (Allan and Poulter, 1992), although some of the wave energy will undoubtedly be lost in the magnetosphere by local damping processes in the plasma such as Landau damping. This process thus allows the free energy from the energetic ring current to be dissipated into the ionosphere and represents a significant energy sink for this system.

Only values for the energy dissipated into the ionosphere can be directly compared to previous publications, as there are none which the authors are aware which indicate the free energy available at the wave source. Greenwald and Walker (1980) calculated that an externally driven large-scale Pc5 wave dissipated $\sim 10^{13} \mathrm{~J}$ of energy in the ionosphere. Large-scale waves, which are sustained by an external energy source, such as the Kelvin-Helmholtz instability or solar wind buffeting, clearly involve larger energies than the internally driven waves examined here.

Allan and Poulter (1984) reported observations of a storm time Pc5 pulsation and also a Pg using the STARE radar, both wave types being driven by wave-particle interactions. They estimated that $\sim 10^{10} \mathrm{~J}$ dissipated into the ionosphere for the storm time Pc5; identical to the estimate made here for the wave detailed in Sect. 4.1. In the case of the Pg, they also infer that the wave was the result of an even mode standing wave interacting through a drift-bounce resonance interaction with $10 \mathrm{keV}$ protons, the same mechanism as proposed for the Pg detailed in case 2 , and also ascertained that $\sim 10^{11} \mathrm{~J}$ was dissipated in the ionosphere, a value consistent with the calculations undertaken in this paper.

It is illuminating to compare the two studies presented above, with the statistical study of the driving magnetospheric particle populations which was the subject of a paper by Baddeley et al. (2005). This paper statistically analysed the particle population database described in this paper in Sect. 4.1. The data were binned according to MLT location and also according to the free energy each contained in the form of a positive gradient region in the IDF. Some of the results from Baddeley et al. (2005) are shown in Fig. 9. The radial distance of the semi-circle indicates increasing amounts of free energy available, from $\sim 1 \times 10^{10} \mathrm{~J}$ to $\sim 2 \times 10^{11} \mathrm{~J}$. The data bins are colour coded as a percentage occurrence in that MLT bin. The results imply that at pre-noon MLTs, the amount of free energy is such that high $m$ waves can frequently be generated via wave particle interactions, if the resonance condition matches a field line eigenmode. 


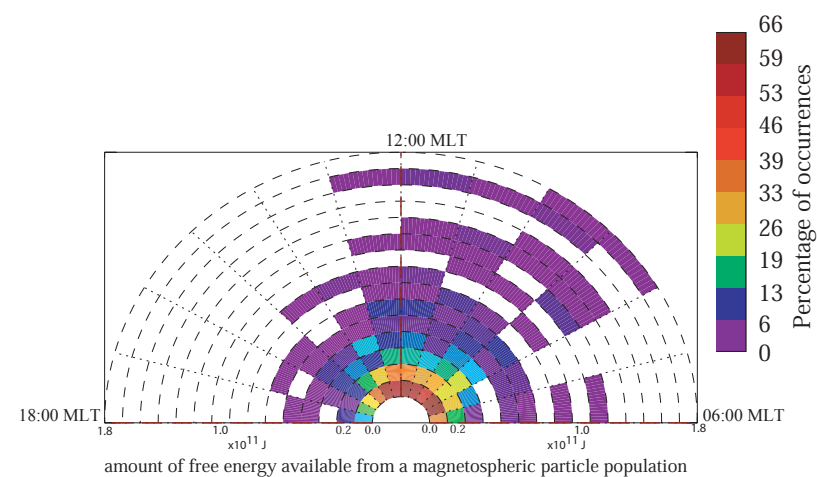

Fig. 9. (Modified from Fig. 5 taken from Baddeley et al., 2005). Results from a statistical study of magnetospheric particle populations, using the TIMAS and CAMMICE (MICS) instruments. Almost three years of data were binned according to MLT location and also according to the free energy each IDF contained in the form of a positive gradient region. The radial distance from the centre of the semi-circle indicates increasing amounts of free energy available up to a maximum of $1.8 \times 10^{11} \mathrm{~J}$ in bin increments of $1.2 \times 10^{10} \mathrm{~J}$. The data bins are colour coded as a percentage occurrence in that MLT bin.

However, when considering Pgs, the results suggest that even if the resonance conditions are met, they will be a rare, morning phenomena due to the small statistical probability of a particle population containing enough free energy to drive the wave. The results from Baddeley et al. (2005) also suggests that the conjugate existence of an IDF containing not only a positive gradient region at the correct particle energy, but also enough free energy to drive the Pg (Wright et al., 2001) is very unlikely to be coincidental given the small probability of such an IDF existing in the magnetospheric particle population. This additional statistical evidence strongly supports the work of Wright et al. (2001) that the $\sim 10 \mathrm{keV}$ particle population was indeed the driving source of the Pg via the drift-bounce resonance interaction. Detailed statistics of the particle distribution functions over all local times are presented in Baddeley et al. (2005).

\section{Summary}

The approach taken in this study demonstrates quantitatively how important ULF waves are when considering energy transport from the magnetospheric ring current into the highlatitude ionosphere. Firstly, this paper has presented a Monte Carlo statistical study to test the cause and effect relationship between the free energy possessed by driving magnetospheric particle populations and conjugate ULF wave signatures observed in the ionosphere. The analysis indicated that the average free energy available from a particle population during times when a high $m$ wave is observed in the conjugate ionosphere is statistically greater than that seen at random. The case studies presented in this paper additionally indicate that the amount of free energy available can possibly determine the magnitude of the wave signature in the ionosphere. We have shown that 10 times more energy is transferred from the magnetospheric particle population into the ionosphere when considering a larger amplitude high $m$ ULF wave; a Pg. The particle statistical study also indicates that even if the resonance condition is met and free energy is available to the wave field, Pgs are likely to be a rare morning phenomenon due to the larger energy requirements of this type of wave. Further investigation using conjugate waveparticle observations is clearly needed in order to develop a better understanding into the nature and magnitude of this energy transfer process.

Acknowledgements. The authors would like to thank T. Fritz, PI of CAMMICE (MICS), W. Peterson, PI of TIMAS and the Royal Society and PPARC for funding the DOPE project. LJB is supported by PPARC.

Topical Editor T. Pulkkinen thanks A. D. M. Walker and another referee for their help in evaluating this paper.

\section{References}

Allan, W. and Poulter, E. M.: ULF waves - their relationship to the structure of the Earth's magnetosphere, Rep. Prog. Phys., 55, 533-598, 1992.

Allan, W. and Poulter, E. M.: The spatial structure of different ULF pulsation types: A review of STARE radar results, Rev. Geophys. and Space Phys., 22, 85-7, 1984.

Baddeley, L. J., Yeoman, T. K., Wright, D. M., Davies, J. A., Trattner, K. J., and Roeder, J. L.: Morning sector drift-bounce resonance driven ULF waves observed in artificially induced HF radar backscatter, Ann. Geophys., 20, 1487-1498, 2002,

SRef-ID: 1432-0576/ag/2002-20-1487.

Baddeley, L. J., Yeoman, T. K., Wright, D. M., Trattner, K. J., and Kellet, B. J.: A Statistical study of unstable particle populations in the global ring current and their relation to the generation of high $m$ ULF waves, Ann. Geophys., in press, 2005.

Cheng, C. Z.: Kinetic - Theory of collisionless ballooning modes, Phys. Fluids, 25, 1020-1026, 1982.

Cheng, C. Z. and Qian, Q.: Theory of Ballooning - Mirror instabilities for anisotropic pressure plasmas in the magnetosphere, J. Geophys. Res., 99, 11 193-11 209, 1994.

Chisham, G.: Giant pulsations: An explanation for their rarity and occurrence during geomagnetically quiet times, J. Geophys. Res., 101, 24 755-24 763, 1996.

Chisham, G. and Orr, D.: Statistical studies of giant pulsations (Pgs): harmonic mode, Planet. Space Sci., 39, 999-1006, 1991.

Chisham, G., Orr, D., and Yeoman, T. K.: Observations of a giant pulsation $(\mathrm{Pg})$ across an extended array of ground magnetometers and an auroral radar, Planet. Space Sci., 40, 953-964, 1992.

Dungey, J. W.: The Structure of the exosphere or Adventures in velocity space, Geophysics, The Earth's Environment, C. Dewitt, Ed., 537, Gordon and Breach, New York, 1963.

Glassmeier, K. H.: Magnetometer Array Observations of a Giant Pulsation Event, J. Geophys. Res., 48, 138-147, 1980.

Glassmeier, K. H., Buchert, S., Motschmann, U., Korth. A, and Pedersen, A.: Concerning the generation of geomagnetic giant pulsations by drift-bounce resonance ring current instabilities, Ann. Geophys., 17, 338-350, 1999,

SRef-ID: 1432-0576/ag/1999-17-338. 
Greenwald, R. A. and Walker, A. D. M.: Energetics of long period resonant hydromagnetic waves, Geophys. Res. Lett., 7, 745-748, 1980.

Hasegawa, A.: Drift mirror instability in the magnetosphere, Phys. Fluids, 12, 2642-2650, 1969.

Hasegawa, A.: Drift - wave instabilities of a compressional mode in high $-\beta$ plasma, Phys. Rev. Lett., 27, 11-14, 1971.

Hughes, W. J.: Hydromagnetic waves in the magnetosphere, Solar Terrestrial Physics (edited by Carovillano, R. L. and Forbes, J. M.), Reidel, Dordrecht, 1983.

Hughes, W. J. and Southwood, D. J.: The screening of micropulsation signals by the atmosphere and ionosphere, J. Geophys. Res., 81, 3234-3240, 1976.

Hughes, W. J., Southwood, D. J., Mauk, B. H., Mcpherron, R. L., and Barfield, J. N.: Alfvén waves generated by an inverted plasma energy distribution, Nature, 275, 43, 1978.

Kivelson, M. G. and Southwood, D. J.: Charged particle behaviour in low frequency geomagnetic pulsations, 4. Compressional Waves, J. Geophys. Res., 90, 1486-1498, 1985.

Kokubun, S., Erickson, K. N., Fritz, T. A., and McPherron, R. L.: Local time asymmetry of Pc4-5 pulsations and associated particle modulations at synchronous orbit, J. Geophys. Res., 94, 6607-6625, 1989.

Newton, R. S., Southwood, D. J., and Hughes, W. J.: Damping of geomagnetic pulsations by the ionosphere, Planet. Space Sci., 26, 201-209, 1978.

Pokhotelov, O. A., Pilipenko, V. A., Nezlina, Y. M., Woch, J., Kremser, G., North, A., and Amata, E.: Excitation of high $\beta$ plasma instabilities at the geostationary orbit - theory and observations, Planet. Space Sci., 34, 695-712, 1986.

Shelley, E. G., Ghielmetti, A. G., Balsiger, H., Black, R. K., Bowles, J. A., Bowman, R. P., Bratschi, O., Burch, J. L., Carlson, C. W., Coker, A. J., Drake, J. F., Fischer, J., Geiss, J., Johnstone, A., Kloza, D. L., Lennartsson, O. W., Magoncelli, A. L., Paschmann, G., Peterson, W. K., Rosenbauer, H., Sanders, T. C., Steinacher, M., Walton, D. M., Whalen, B. A., and Young, D. T.: The Toroidal Imaging Mass-Angle Spectrograph (TIMAS) for the Polar Mission, Space Sci. Rev., 71, 497-530, 1995.
Southwood, D. J.: A general approach to low-frequency instability in the ring current plasma, J. Geophys. Res., 81, 3340-3348, 1976.

Southwood, D. J., Dungey, J. W., and Etherington, R. J.: Bounce resonant interactions between pulsations and trapped particles, Planet. Space Sci., 17, 349-361, 1969.

Thompson, S. M. and Kivelson, M. G.: New Evidence for the origin of giant pulsations, J. Geophys. Res., 106, 21 237-21 253, 2001.

Wilken, B., Weiß, W., Hall, D., Grande, M., Sørass, F., and Fennell, J. F.: Magnetospheric Ion Composition Spectrometer on board the CRRES spacecraft, J. Spacecraft and Rockets, 29, 585-591, 1992.

Wright, D. M. and Yeoman, T. K.: High-latitude HF Doppler observations of ULF waves: 2 . Waves with small spatial scale sizes, Ann. Geophys., 17, 868-876, 1999,

SRef-ID: 1432-0576/ag/1999-17-868.

Wright, D. M., Yeoman, T. K., and Chapman, P. J.: High-latitude HF Doppler observations of ULF waves: 1 . waves with large spatial scale sizes, Ann. Geophys., 15, 1548-1556, 1997,

SRef-ID: 1432-0576/ag/1997-15-1548.

Wright, D. M., Yeoman, T. K., and Davies, J. A.: A comparison of EISCAT and HF Doppler observations of a ULF wave, Ann. Geophys., 16, 1190-1199, 1998,

SRef-ID: 1432-0576/ag/1998-16-1190.

Wright D. M., Yeoman, T. K., Rae, I. J., Storey, J., Stockton-Chalk, A. B., Roeder, J. L., and Trattner, K. J.: Ground-based and Polar spacecraft observations of a giant $(\mathrm{Pg})$ pulsation and its associated source mechanism, J. Geophys. Res., 106, 10 837-10 852, 2001.

Yeoman, T. K. and Wright, D. M.: High-latitude Observations of ULF Waves with Large Azimuthal Wavenumbers, J. Geophys. Res., 105, 5453-5462, 2000

Yeoman, T. K., Wright, D. M., Robinson, T. R., Davies, J. A., and Rietveld, M.: High spatial and temporal resolution observations of an impulse-driven field line resonance in radar backscatter artificially generated with the Troms $\varnothing$ heater, Ann. Geophys., 15, 634-644, 1997,

SRef-ID: 1432-0576/ag/1997-15-634. 